\title{
Changes to near-surface region of PTFE during dry sliding against steel
}

\author{
S. K. BISWAS \\ Department of Mechanical Engineering, Indian Institute of Science, Bangalore 560 012, India \\ KALYANI VIJAYAN \\ Materials Science Division, National Aeronautical Laboratory, Bangalore 560 017, India
}

PTFE specimens were slid against an EN24 disc. The unworn and worn surfaces as well as the wear debris were examined by X-ray diffraction. Sliding was found to introduce (i) shrinkage of the unit cell, (ii) enlargement of crystallites and (iii) residual stresses in the slid PTFE surface. No conformational changes in the 15, helix could be observed due to sliding. The wear debris was found to be $1 \mu \mathrm{m}$ thick warped laminates.

\section{Introduction}

Polytetrafluoroethylene (PTFE) is an important bearing material. Under most practical conditions its friction coefficient is observed to be low and the wear rate high as compared to other polymers. The latter has prompted many mechanistic investigations.

In sliding PTFE against itself and glass, Makinson and Tabor [1] found two regimes of friction related to temperature and sliding speed. They proposed that in the low-friction regime crystalline slices shear against each other, a slice becoming a thin film which is transferred to the counterface. Under high-friction conditions the viscoelastic properties dictate an increase in the shear strength and the shear takes place between crystallites and grains, leading to lumpy transfer of debris. Later work by Pooley and Tabor [2], however, challenged the low-friction hypothesis as the transferred film was estimated to be of the order of $2 \mathrm{~nm}$, which is substantially less than the $20 \mathrm{~nm}$ crystalline slice thickness. Tanaka et al. [3], however, find the film to be of the order of $30 \mathrm{~nm}$ thick and propose that the transferred film consists of slices from adjacent crystalline slices which join each other edge-on under sliding conditions. They explain that thick debris fragments are nothing but a number of films which pile up on each other.

McLaren and Tabor [4], found the friction characteristics of PTFE to be viscoelastically sensitive. This lends credibility to the above model, which proposes the basic shearing to be taking place in the viscoelastically sensitive amorphous regime separating two crystalline slices. Tanaka et al. [3] found the wearsliding speed specimen temperature characteristics to change at about $100^{\circ} \mathrm{C}$, a temperature close to the 127 "C transition in viscoelastic or internal friction properties, observed for PTFE by McCrum [5].

The direct effect of structural parameters such as crystallinity and band width (which could be expected to alter the viscoelastic properties) on the tribological characteristics of PTFE is however inconclusive. Tanaka et al. [3] report that it is only the band width and not the crystallinity which affects the wear resistance of PTFE. Lontz and Kumnick [6] report the wear resistance to improve with increasing crystallinity. This is supported by the work of Briscoe and Zhilong [7] who found $\gamma$-irradiation of PTFE to improve its wear resistance as it increased its crystallinity.

PTFE is known to undergo viscoelastic/internal friction transitions at temperatures of 19 to $20^{\circ} \mathrm{C}$, $127^{\prime \prime} C\left(T_{\mathrm{g}}\right)$ and $327^{\prime \prime} C\left(T_{\mathrm{m}}\right)$. At 20 and $30^{\prime \prime} C$ PTFE undergoes a first-order crystalline phase transition with an increment of 1 to $1.3 \%[5,8]$ in specific volume. X-ray diffraction studies have shown the PTFE unit cell to be triclinic up to $20^{\circ} \mathrm{C}$. The molecular conformation is a $13_{6}$ helix with a $180^{\circ}$ twist with a repeat distance of $1.68 \mathrm{~nm}$ consisting of $13 \mathrm{CF}_{2}$ groups [9]. At $20^{\circ} \mathrm{C}$ a slight untwisting of the spiral occurs and the molecular conformation changes to 15 , helix with repeat distance of $1.95 \mathrm{~nm}$ [10]. Above 20 " $\mathrm{C}$ the molecular packing becomes hexagonal and remains unchanged even after the 30 " $\mathrm{C}$ transition. In the present investigation PTFE specimens are slid against EN24 steel discs. A range of normal load, speed and sliding distances are chosen to produce frictional heat which takes the starting interface temperature of 21.5 " C past 30 " $C$ with sliding. The wear, friction and interface temperature characteristics are observed. X-ray diffraction studies are conducted on $3 \mathrm{~mm}$ slices, one side of which are the worn interfaces with wear debris. Differential scanning calorimetry (DSC) studies are carried out using a $5 \mathrm{mg}$ sample. The aim of the investigation was to study possible structural changes in PTFE introduced by the sliding process, changes which may have a bearing on the wear mechanism of this common bearing material.

\section{Experimental details}

A pin-on-disc machine was used for conducting sliding experiments in a range of 0.06 to $0.42 \mathrm{MPa}$ normal pressure and 0.1 to $1.2 \mathrm{~m} \mathrm{sec}^{-1}$ surface speed. PTFE cylinders of $6 \mathrm{~mm}$ diameter and $10 \mathrm{~mm}$ length were machined from commercially supplied $16 \mathrm{~mm}$ 
diameter rods. The flat ends of the PTFE cylinders were slid against EN2 4 discs, hardened and ground to a surface finish of $0.3 \mu \mathrm{m}$ centre line average (cla). Wear was measured in situ by linear voltage differential transformers (LVDT) attached to the pin arm, and friction force by a load cell bearing against the pin holder.

\subsection{Temperature measurement}

The sliding interface temperature was estimated by polynomial extrapolation of the readings obtained from three thermocouples (Alumel-Chromel, 40 gauge) placed $2 \mathrm{~mm}$ apart from each other, the first one being at a distance of $2 \mathrm{~mm}$ from the interface. Thermocouples were inserted into $1 \mathrm{~mm}$ diameter drilled radial holes.

\subsection{Density measurements}

The weight-loss method was used to measure the density of the bulk sample, which was found to be $\mathbf{2 . 2 8}$.

The percentage of the crystalline fraction $x_{\mathrm{c}}$ of the PTFE unworn sample was determined using the following relation [11]:

$$
x_{\mathrm{c}}=100 \frac{\varrho_{\mathrm{c}}\left(\varrho-\varrho_{\mathrm{a}}\right)}{\varrho\left(\varrho_{\mathrm{c}}-\varrho_{\mathrm{a}}\right)}=82.46 \%
$$

where $\varrho_{\mathrm{c}}=2.35$, the reported [12] density of $100 \%$ crystalline PTFE; $\varrho_{\mathrm{a}}=2.00$, the reported [12] density of a $100 \%$ amorphous PTFE; and $\varrho=2.28$, the measured density of the semicrystalline PTFE.

\subsection{X-ray diffraction}

$\mathrm{X}$-ray diffraction patterns of the unworn and worn PTFE specimens were recorded on a Philips powder diffractometer, using copper $K \alpha$ radiation and a proportional counter. The samples were rotated at a speed of either $1 / 4$ or $1 / 8^{\circ} \mathrm{min}^{-1}$ and the chart speed was chosen at $20 \mathrm{mmmin}^{-1}$. The specimen was prepared by cutting a $3 \mathrm{~mm}$ thick slice from the worn end of the PTFE cylinder. The worn side of the disc was exposed to the $\mathrm{X}$-rays.

The peak positions of the reflections were determined by taking the mid-points of lines drawn parallel to the background level and extrapolating to the maximum. The unit cell dimensions were determined by least-squares refinement using all the observed reflections. The integrated intensities were obtained by estimating the area under each profile.

\subsection{Differential scanning calorimetry}

A Perkin-Elmer DSC 2 was used to characterize and measure the thermal properties of PTFE. The endotherm peaks of the phase transition were recorded at a scanning rate of $10^{\circ} \mathrm{min}^{-1}$ and a sensitivity of $10 \mathrm{mcal} \mathrm{sec}^{-1}\left(42 \mathrm{~mJ} \mathrm{sec}{ }^{-1}\right)$. Areas under each endotherm profile of the bulk PTFE and debris were measured to calculate the relative crystallinity values.

\section{Results}

Fig. 1 is a typical record showing the variation of wear rate, friction coefficient and (estimated) interface temperature with sliding distance. It is seen that while the wear rate remains approximately at a level of $1 \mathrm{x}$ $10^{-8} \mathrm{~cm}^{3} \mathrm{~cm}^{-1}$ up to a sliding distance of $0.18 \mathrm{~km}$, a short distance from this the wear rate jumps threefold. Beyond $0.22 \mathrm{~km}$, the average level of wear rate remains at about $3 \times 10^{-8} \mathrm{~cm}^{3} \mathrm{~cm}^{-1}$. The same trend appears to prevail for the estimated interface temperature, the

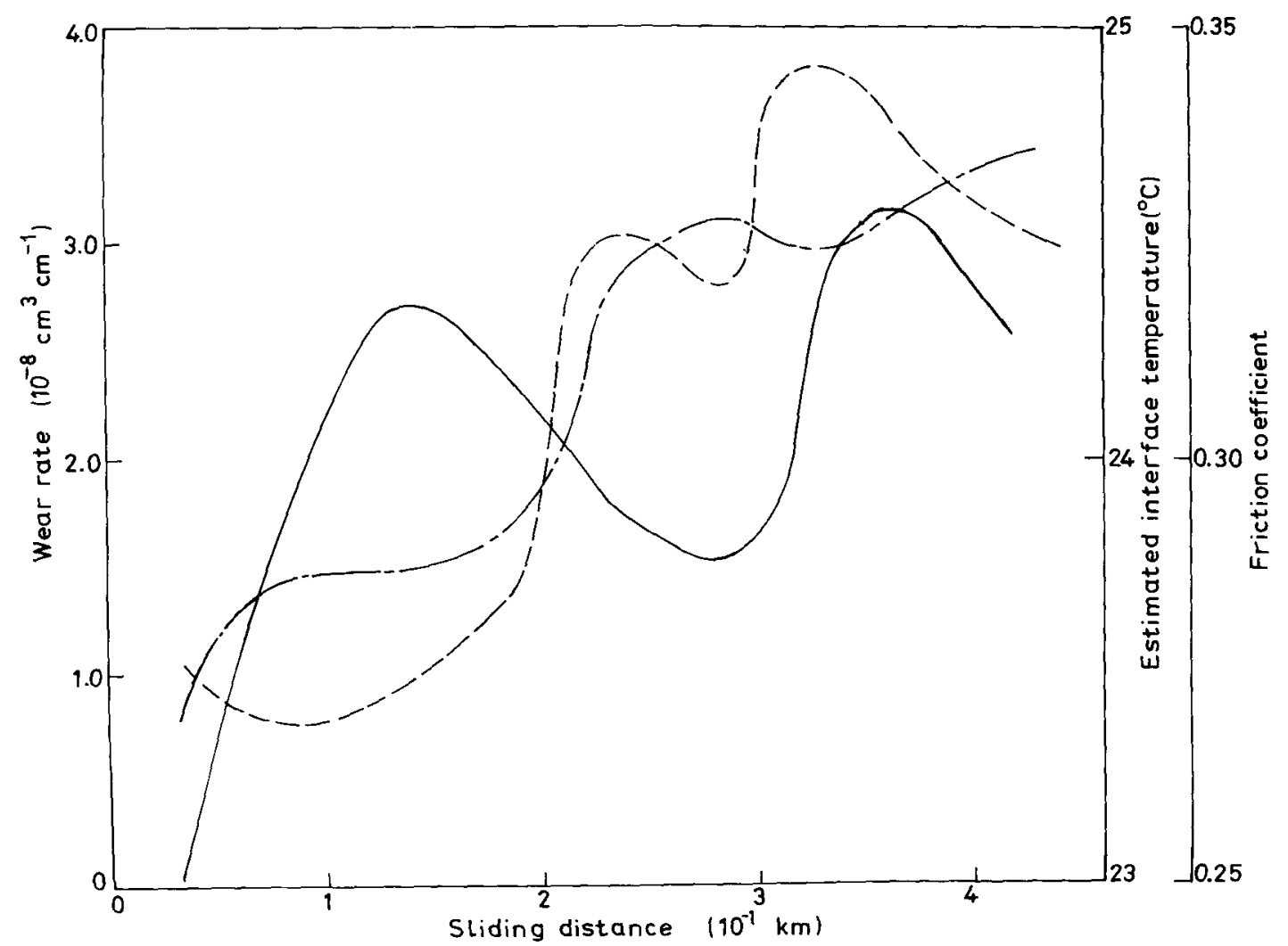

Figure 1 A typical experiment record. Pressure $0.086 \mathrm{MPa}$, speed, $0.3 \mathrm{~m} \mathrm{sec}^{-1}$.(-) Friction coefficient, $(-,-)$ temperature, $(--)$ wear rate. 

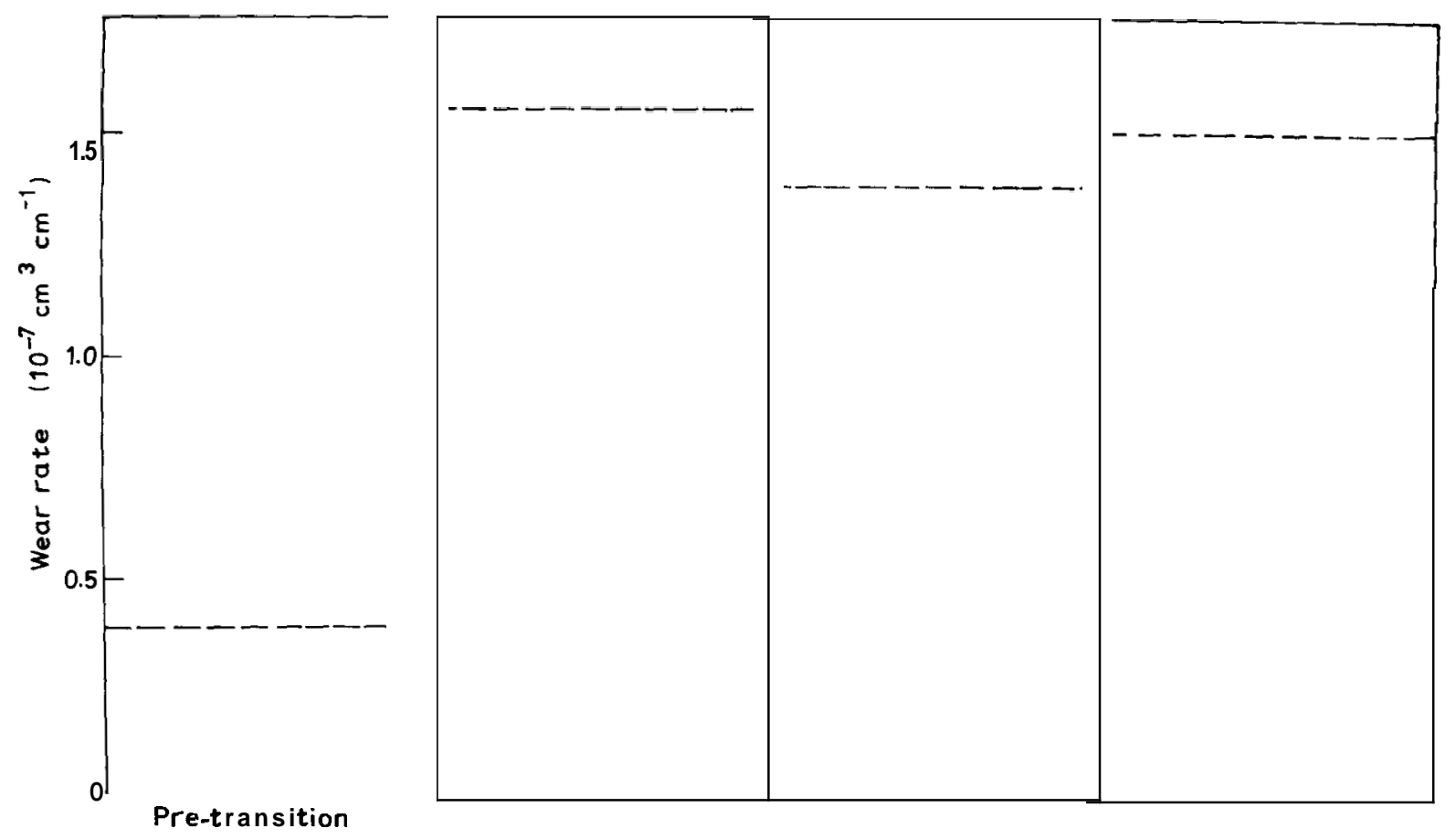

Figure 2 Change in wear rate due to transition. Pressure $0.345 \mathrm{MPa}$, speed $0.24 \mathrm{~m} \mathrm{sec}^{-1}$

initiation of the temperature jump in terms of sliding distance coinciding with that of wear rate. This temperature is henceforth called the transition temperature. The coefficient of friction. which increases with sliding distance in the pre-transition stage, oscillates
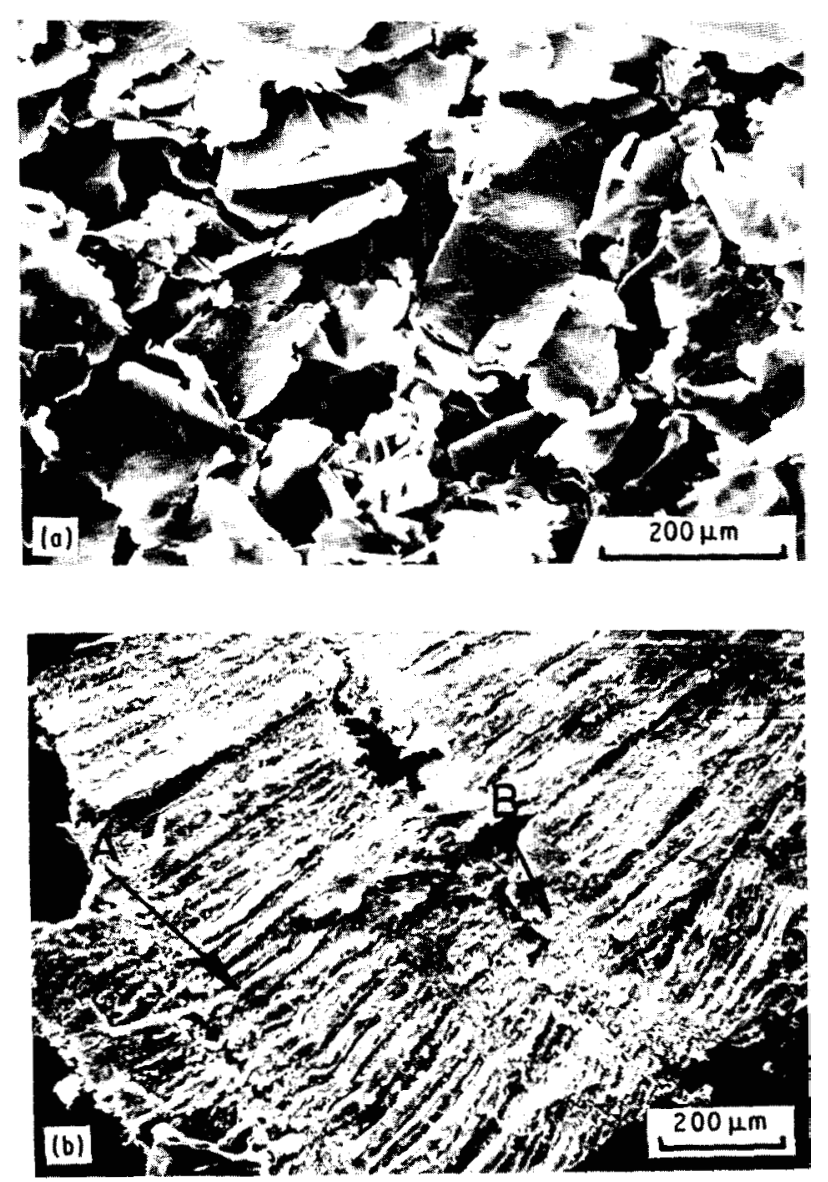

about a mean value after transition. The change in wear rate at the transition is irreversible as can be made out from Fig. 2.

Fig. 3 shows a scanning micrograph of debris collected during the sliding experiments. The transition in wear rate is clcarly reflected (morphologically and dimensionally) by the different types of debris generated in the pre- and post-transition phases. The pre-transition debris consists of thin (I to $2 \mu \mathrm{m}$ ) segregated flakes of an area of the order of $350 \mu \mathrm{m} \mathrm{x}$ $150 \mu \mathrm{m}$ (dimensions estimated from the micrograph). The post-transition debris is also of about the same thickness as the pre-transition debris, but the posttransition debris fragments are much larger in area and highly structured. They consist of regular bands of corrugated but torn (A in Fig. 3b) sheets held together at the edge of relatively unwarped material (B in Fig. 3b).

Figure 3 Scanning electron micrographs of (a) debris, (b) pre-transition and (c) post-transition. Pressure 0.25 MPa, speed $0.2 \mathrm{~m} \mathrm{sec}^{-1}$

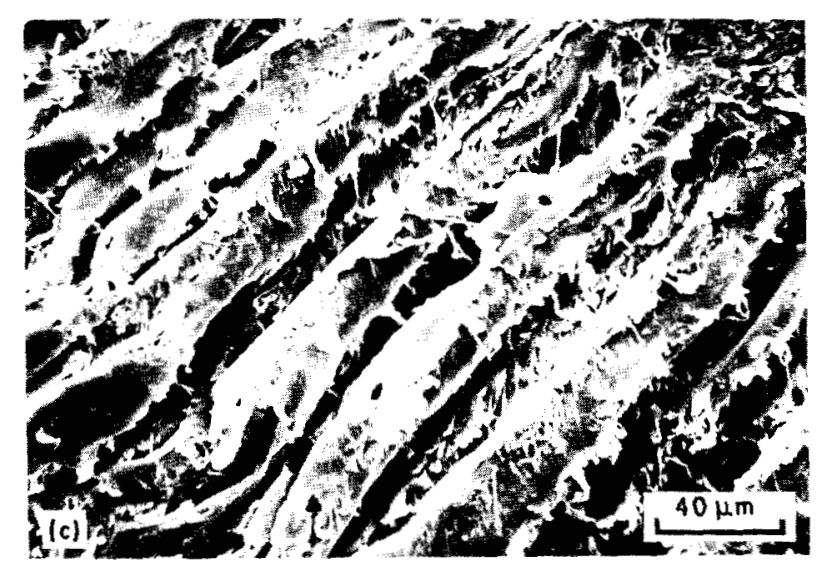




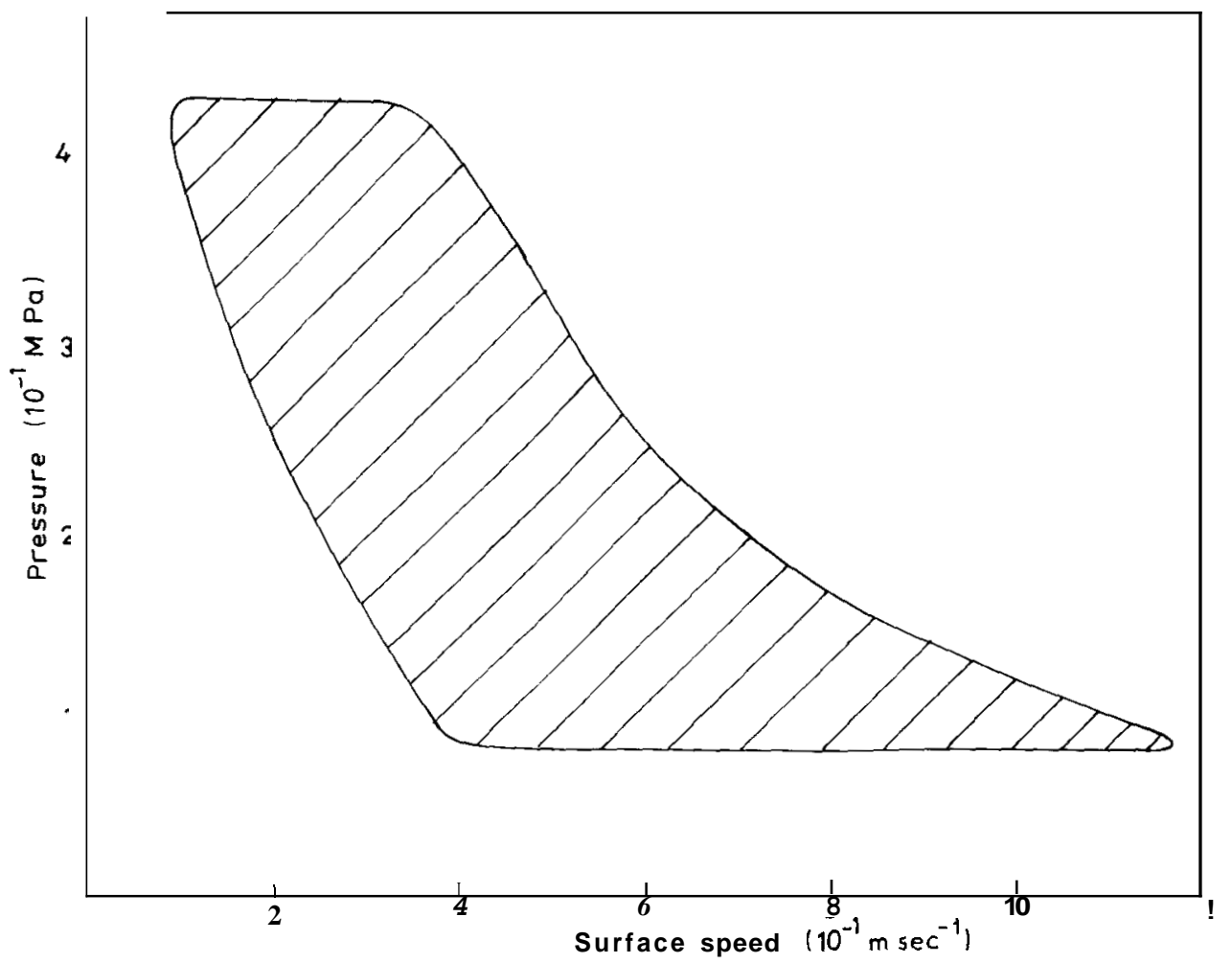

Figure 4 Pressure speed map showing (hatched) the regime where transitions were observed

Over a range of 0.06 to $0.42 \mathrm{MPa}$ bearing pressure and 0.1 to $1.2 \mathrm{~m} \mathrm{sec}^{1}$ sliding speed the transition occurred in the shaded region shown in Fig. 4. Over the unshaded region to the left, pre-transition wear prevailed over a constant interface temperature even when the sliding was continued up to a distance of $10 \mathrm{~km}$. The region to the right of the shaded region exhibited post-transition wear right from the start of the experiment. It is possible that in the latter case the transition occurs in such a short time from the commencement of the experiment that it could not be detected. The size and shape of the region was found to be highly sensitive to environmental temperatures.

Fig. 5 shows the estimated transition temperature (averaged from a result of three identical experiments) as a function of sliding speed (Fig. 5a) and normal load (Fig. 5b). It is seen that transition temperature changes by only about $1{ }^{\circ} \mathrm{C}$ over the test range of pressure and speed, when the environmental temperature is $21.5^{\prime \prime} \mathrm{C}$.

Fig. 6 shows the variation in average pre- $(\mathrm{W}$,$) and$ post- $\left(W_{2}\right)$ transition wear rates as functions of load and speed. $W_{1}$ initially increases and then decreases with sliding speed. $W_{2}$ increases with pressure and decreases with speed. The average coefficients of friction shown in Fig. 7 are post-transition coefficients measured at the end of $4 \mathrm{~km}$ sliding. The coefficient of friction increases with speed and decreases with load.

\subsection{X-ray diffraction}

$\mathrm{X}$-ray diffraction patterns obtained from the unworn and worn PTFE specimens were found to be very similar. Both the sets were characterized by the presence of seven prominent reflections which were identified as $(10.0),(11.0),(10.7),(10.8),(21.0)$, $(00.15)$ and $(10.15)$, respectively, corresponding to the hexagonal unit cell of dimensions $a=0.566 \mathrm{~nm}$ and $c=1.95 \mathrm{~nm}[10]$. However, the diffraction patterns corresponding to the unworn and worn pre- and post-transition specimens differed in details. In particular, the positions of the diffraction maxima $\left(2 \theta_{\max }\right)$, the distribution of intensities $(I)$ and full width at half maximum (FWHM, $b$ ) were found to manifest slight but significant variations.

Table I lists the mean values of the shifts, A, and their respective estimated standard deviations, $\sigma$, averaged

TA B LE I Observed shifts in the unit cell dimensions

\begin{tabular}{lll}
\hline Parameter* & Worn, pre-transition & Worn, post-transition \\
\hline$\langle\Delta a\rangle(\mathrm{nm})$ & 0.00092 & -0.0018 \\
$\sigma(\langle\Delta a\rangle)(\mathrm{nm})$ & 0.00011 & 0.00075 \\
$\langle\langle\Delta a\rangle| / \sigma\langle\Delta a\rangle)$ & 8.4 & $\mathbf{2 . 4}$ \\
$100\langle\Delta a\rangle\langle\langle a\rangle$ (unworn) & 0.16 & -0.31 \\
$\langle\Delta c\rangle(\mathrm{nm})$ & 0.0019 & -0.0055 \\
$\sigma(\langle\Delta c\rangle)(\mathrm{nm})$ & 0.0015 & 0.0010 \\
$\langle\Delta c\rangle \mid / \sigma(\langle\Delta c\rangle)$ & 1.3 & 5.5 \\
$100\langle\Delta c\rangle\langle\langle\mathrm{c})$ (unworn) & 0.1 & -0.28 \\
$100\langle\Delta v\rangle\langle\langle v\rangle$ (unworn) & 0.5 & -1.0 \\
\hline
\end{tabular}

*A refers to (worn unworn). 

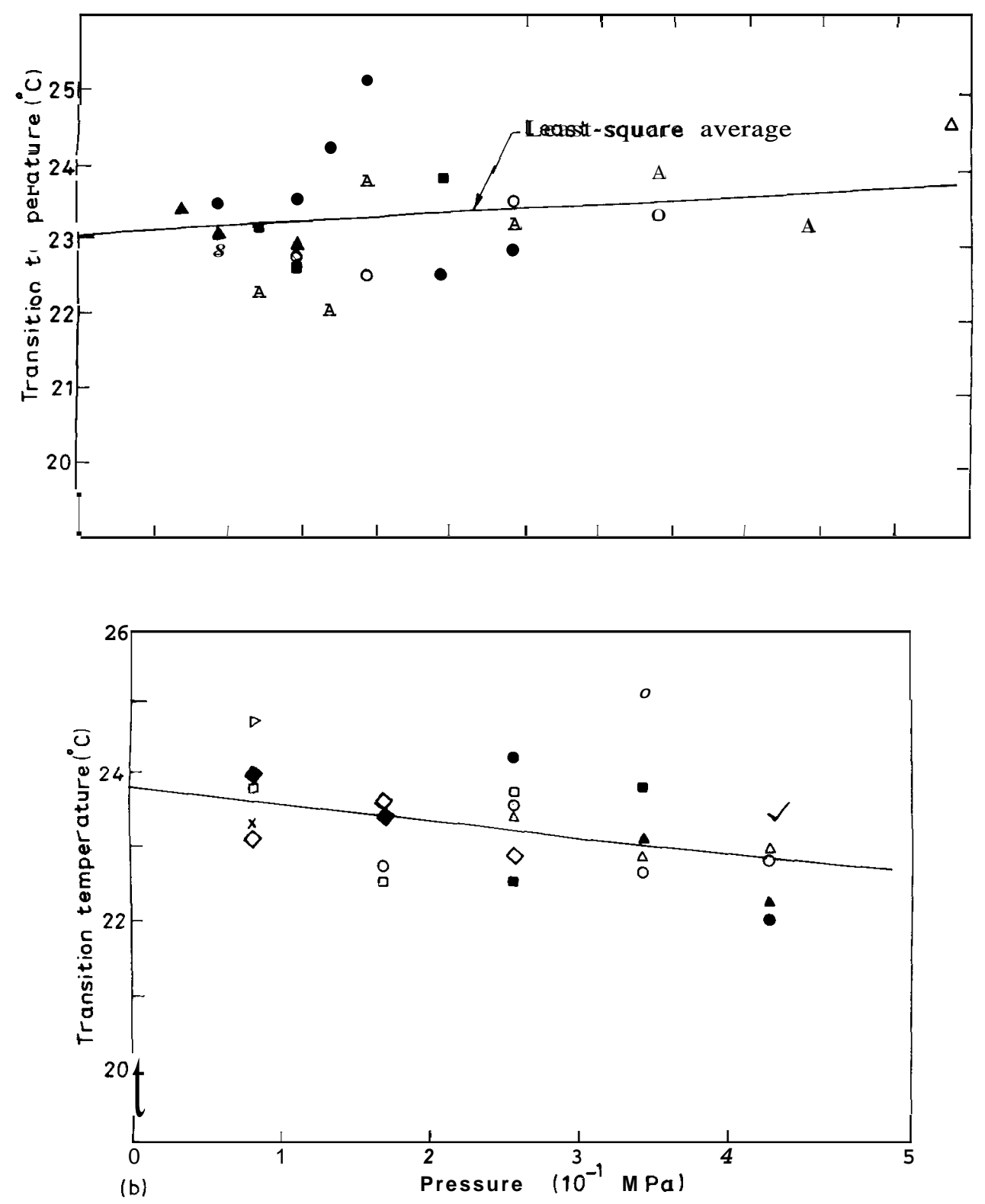

Figure 5 (a)Transition temperature against surface speed. Pressure (MPa): (A) 0.036, (O)0.172, (•) 0.250, (W) 0.344, (A) 0.43. (b) Transition temperature against pressure. Surface speed $\left(\mathrm{m} \mathrm{sec}^{-1}\right):(J) 0.14,(\mathbf{A}) 0.19,(\mathbf{A}) 0.24,(\mathbf{O}) 0.29,(\bullet) 0.34,(\square) 0.39$. (口) $0.48,(\diamond) 0.58,(\bullet) 0.78$. (x) 0.97 , (D) 1.17 .

over three specimens (using the method given by Topping [13]). From the values of $(\Delta a\rangle / \sigma\langle\boldsymbol{A} \boldsymbol{u})$ and $\langle\Delta c\rangle / \sigma\langle\mathbf{A c})$ it may be noted that the observed mean shifts, though small in magnitude, are statistically significant. Comparison of the mean shifts shows that in both the worn pre- and post-transition states, the percentage changes in the $a$ and $\mathrm{c}$ dimensions are comparable. In the worn pre-transition state, both $a$ and $c$ lengths exhibit slight enlargement, leading to an

T A B $L$ E I I Observed variation in the normalized intensities

\begin{tabular}{lll}
\hline$h k . l$ & \multicolumn{2}{l}{$=I(h k . l) / I(10.0)$} \\
\cline { 2 - 3 } & Pre-transition & Post-transition \\
\hline 11.0 & 1.15 & 0.61 \\
10.7 & 0.89 & 1.07 \\
10.8 & 1.06 & $1.1 \mathrm{I}$ \\
21.0 & 0.80 & 0.79 \\
00.15 & 1.04 & 0.85 \\
10.15 & 1.14 & 0.85 \\
\hline
\end{tabular}

increase in the unit cell volume, $v$, by $\simeq 0.5 \%$. In contrast, in the worn post-transition state, both the axial lengths are found to decrease and a consequent reduction in the unit cell volume by $\simeq 1.1 \%$ is observed.

In Table II, average values of the normalized intensities $\boldsymbol{R}=I(h k l) / I(10.0)$ are listed.

The observed variations in the $b$ values with sliding are presented in Table III. The feature to be noted is that the profiles do not exhibit broadening due to sliding but tend to sharpen in both the pre- and posttransition states. On account of the large $\sigma$ values, the shifts $(\boldsymbol{A} \boldsymbol{h})$ due to sliding could be considered significant only for the $(21.0)$ and $(00.15)$ reflections in the post-transition state.

\subsection{Wear debris}

The diffraction pattern from the debris is also characterised by the presence of reflections corresponding to the 15 helix. However, the intensities of all the reflections other than $(10.0)$ are very low. The least- 

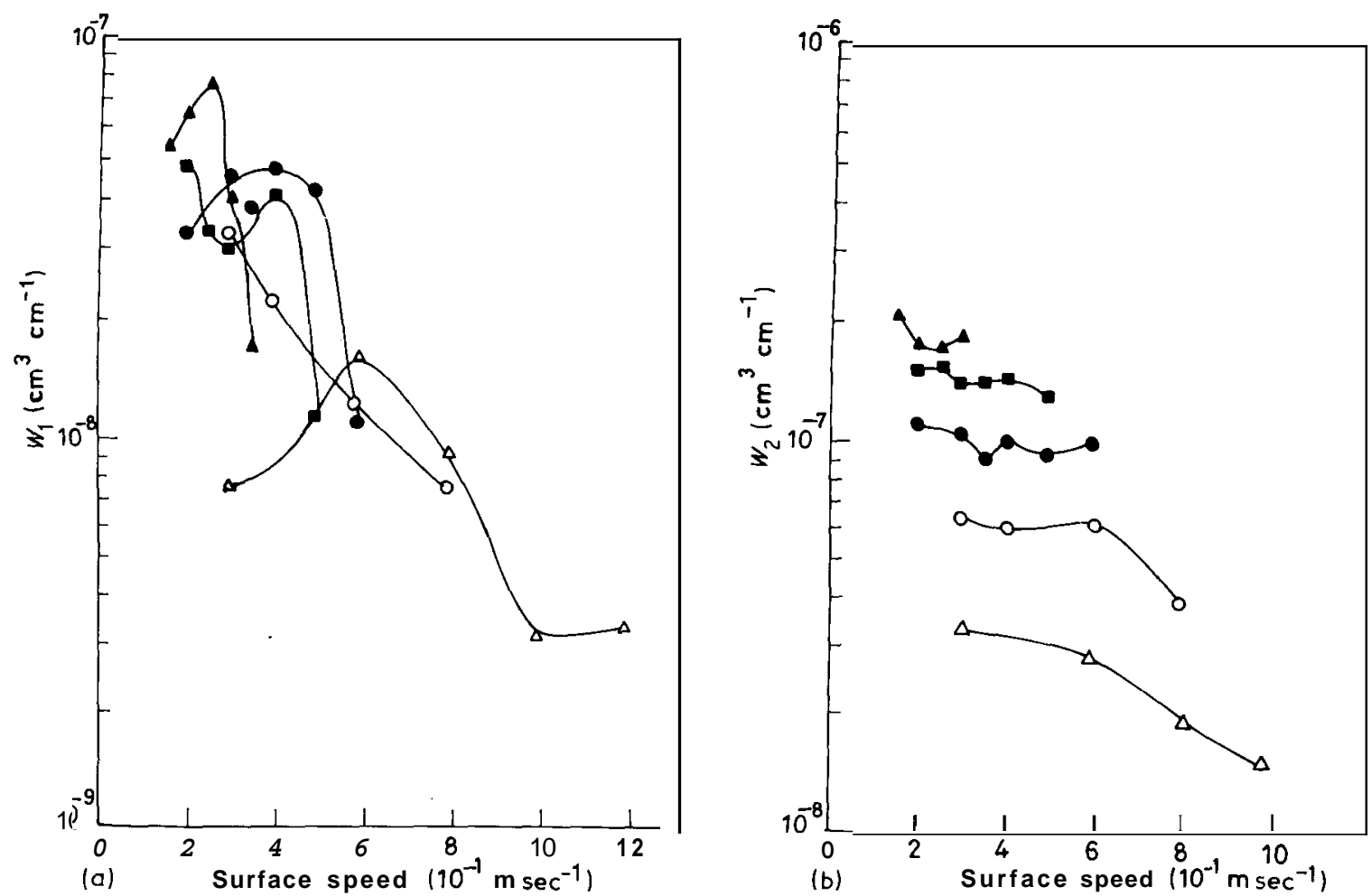

Figure 6 Wear rate against surface speed: (a) pre-transition, (b) post-transuion. Pressure (MPa): (A) 0.086, (O)0.172, (O)0.258, (ם) 0.344, (A) 0.434

squares-refined cell parameters of the debris indicate an increase in the cell volume by $3 \%$ over that corresponding to the post-transition slice. It is also found that the FWHM values of all the reflections are at least three times larger than the corresponding values of the post-transition slice. A typical example is shown in Fig. 8 where the profile of the $(10.0)$ reflection corresponding to the debris is compared with that of the post-transition slice.

\section{Discussion}

In sliding PTFE samples against steel, the rate of wear was found to increase sharply after a certain sliding distance. The estimated temperature of the specimen sliding surface at the transition was found to decrease with normal load and increase with sliding speed. It must be noted that these temperature values are based on extrapolation and should therefore be regarded as only estimates.

Occurrence of the same set of reflections in the patterns from the unworn and worn specimens indi-

TA B LE I I I Observed variations in the FWHM values*

\begin{tabular}{lllll}
\hline$h k . l$ & Pre-transition & \multicolumn{3}{c}{ Post-transition } \\
\cline { 2 - 5 } & $\begin{array}{l}10^{\prime}\langle\Delta b\rangle^{h k \prime} \\
(\mathrm{deg})\end{array}$ & $\frac{|\langle\Delta b\rangle|}{\sigma(\langle\Delta b\rangle)}$ & $\begin{array}{l}10^{3}(\boldsymbol{A} h)^{\prime \prime} \\
(\mathrm{deg})\end{array}$ & $\frac{|\langle\Delta b\rangle|}{\sigma(\langle\Delta b\rangle)}$ \\
\hline 10.0 & -42 & 0.9 & -6 & 0.2 \\
11.0 & -17 & 0.4 & 0 & 0 \\
10.7 & -25 & 0.5 & -13 & 0.3 \\
10.8 & 0 & 0 & -60 & 1.1 \\
21.0 & -34 & 0.8 & -188 & 2.0 \\
00.15 & $-\mathbf{4 6}$ & 0.4 & -25 & 0.9 \\
10.15 & -42 & 1.1 & -125 & 3.3 \\
\hline
\end{tabular}

*A refers to (worn-unworn) cates that the basic 15 , helical conformation of the PTFE chains reported [10] to be the stable conformation between 20 and $30^{\circ} \mathrm{C}$ does not change as a result of the transition. Also the absence of any diffuseness in the diffraction patterns from the worn specimens rules out the possibility of the introduction of largescale molecular disorder due to sliding.

The distribution of the normalized intensity values corresponding to the unworn and worn states (Table II) are very similar, thereby supporting the contention that the molecular structure is not affected by the process of sliding.

The sharpening of the diffraction profiles (Table III) provides strong evidence for three-dimensional growth of the crystallites. It is however, surprising that the reflection $(00.15)$ corresponding to the $c$ axis is not sharpened whereas the reflection $(10.15)$ exhibits marked sharpening. This feature prompts the consideration of the possibility of the PTFE chains being centred about the $[10.151$ direction and not the $[00.11$ direction.

The growth of the crystallites may have happened through reorientation of randomly oriented crystallites (a band of crystalline slices making up a crystallite) situated near the sliding interface. Tangential forces and frictional heat aid adjacent crystallites (which now contain coplanar crystalline slices) to join edge-on in giving rise to larger crystallites. Under sliding conditions coplanar slices from such crystallites are pressed together as well as drawn together to form laminates which emerge on detachment as debris. Tanaka et al. [3], on the basis of micrographic observations of the worn surface, proposed a model similar to this. This plastically worked surface layer, until it is detached as debris, is a part of the elastically deformed 


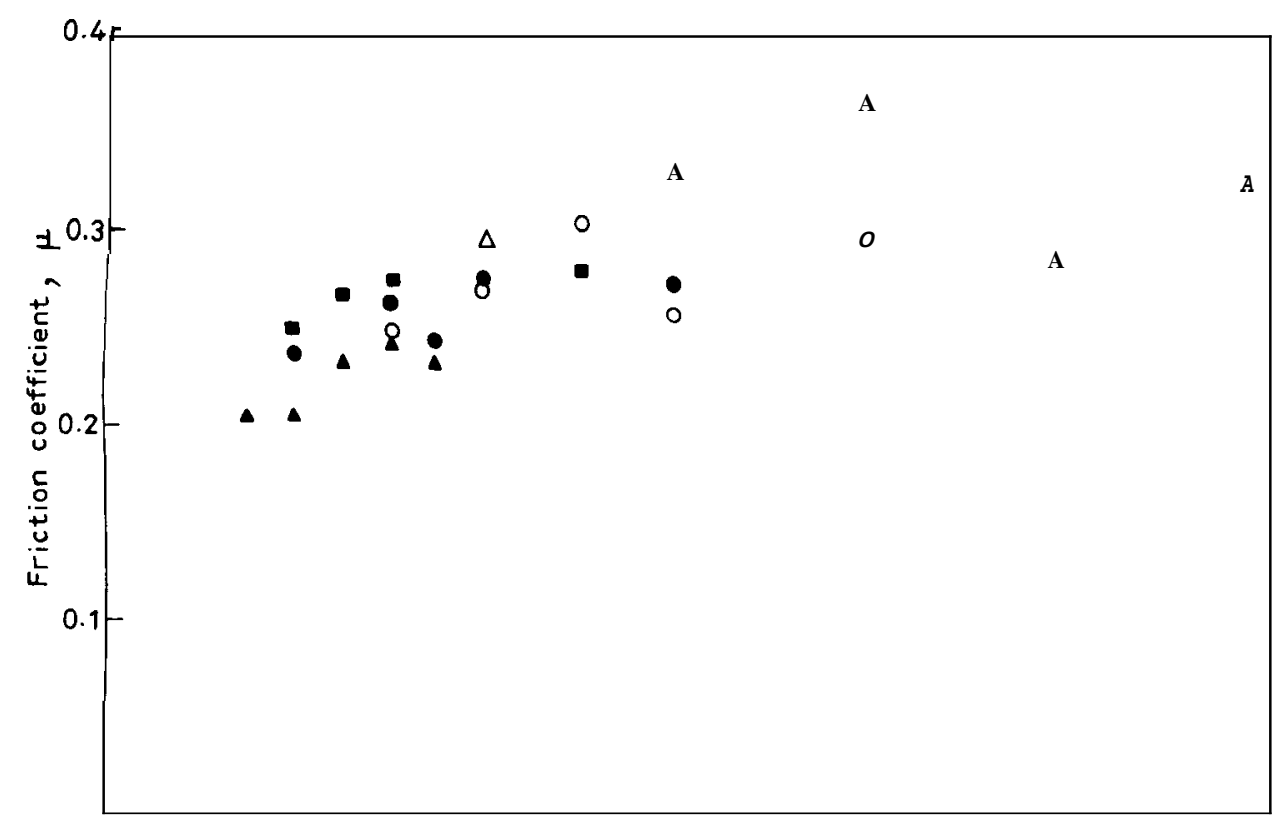

Figure 7 (a) Friction coefficient against surface speed. Pressure (MPa): (A) 0.086, (O) 0.172, (•) 0.256, (匹) 0.344 , (A) 0.430. (b) Friction coefficient against pressure. Surface speed $\left(\mathrm{m} \mathrm{sec}^{-1}\right):(v)$ 0.14 , (A) 0.19. (A) 0.24 , (O) 0.29, (o) 0.34, (O) 0.39, ( $0.48,(\mathrm{O}) 0.58,(\diamond) 0.78,(\mathrm{x})$ 0.97 , (D) 1.17 .

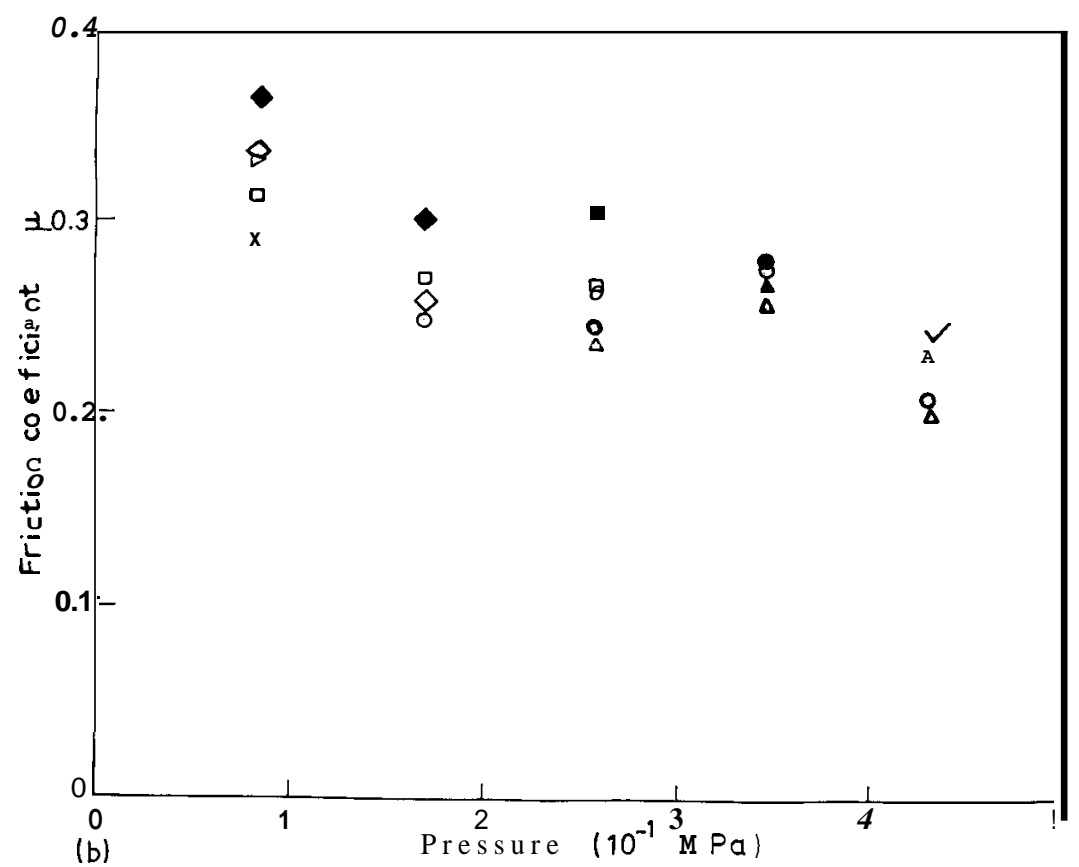

bulk of the PTFE specimen. Thus, when sliding stops, these layers could be expected to develop residual stresses. The presence of residual stresses in these layers was confirmed in the present investigation. A shift was observed in $2 \theta_{\max }$ of the $(00.15)$ diffraction

profile (of the slid surface slice) recorded at $\psi=0$ and $15^{\circ}, \psi$ being the angle between the plane normal and the surface normal. Such a shift is a measure of the residual stress [14].

The comparable fractional changes in the $a$ and

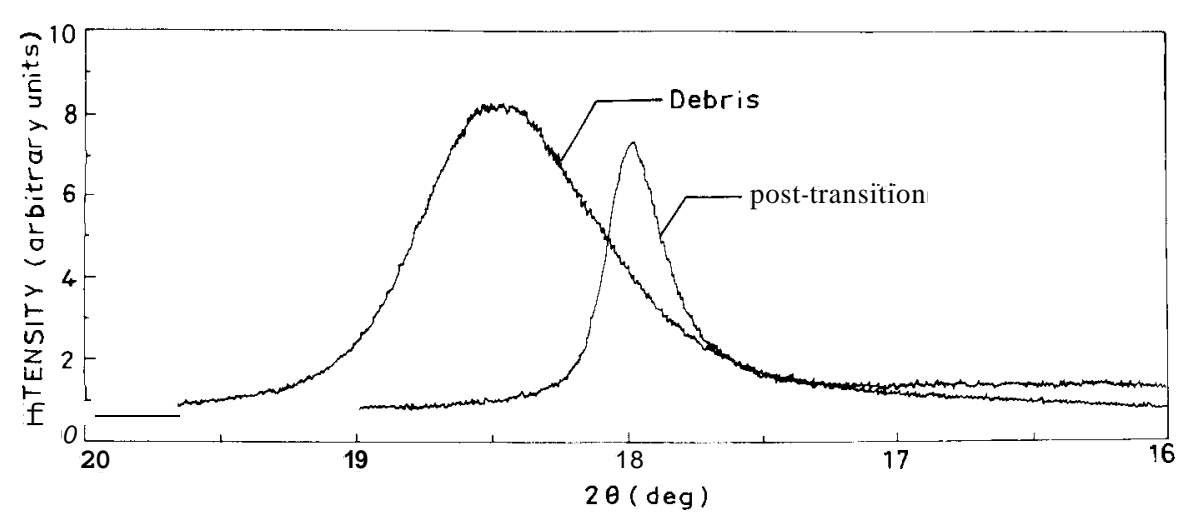

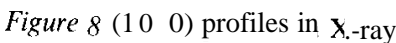
diffractogram: 


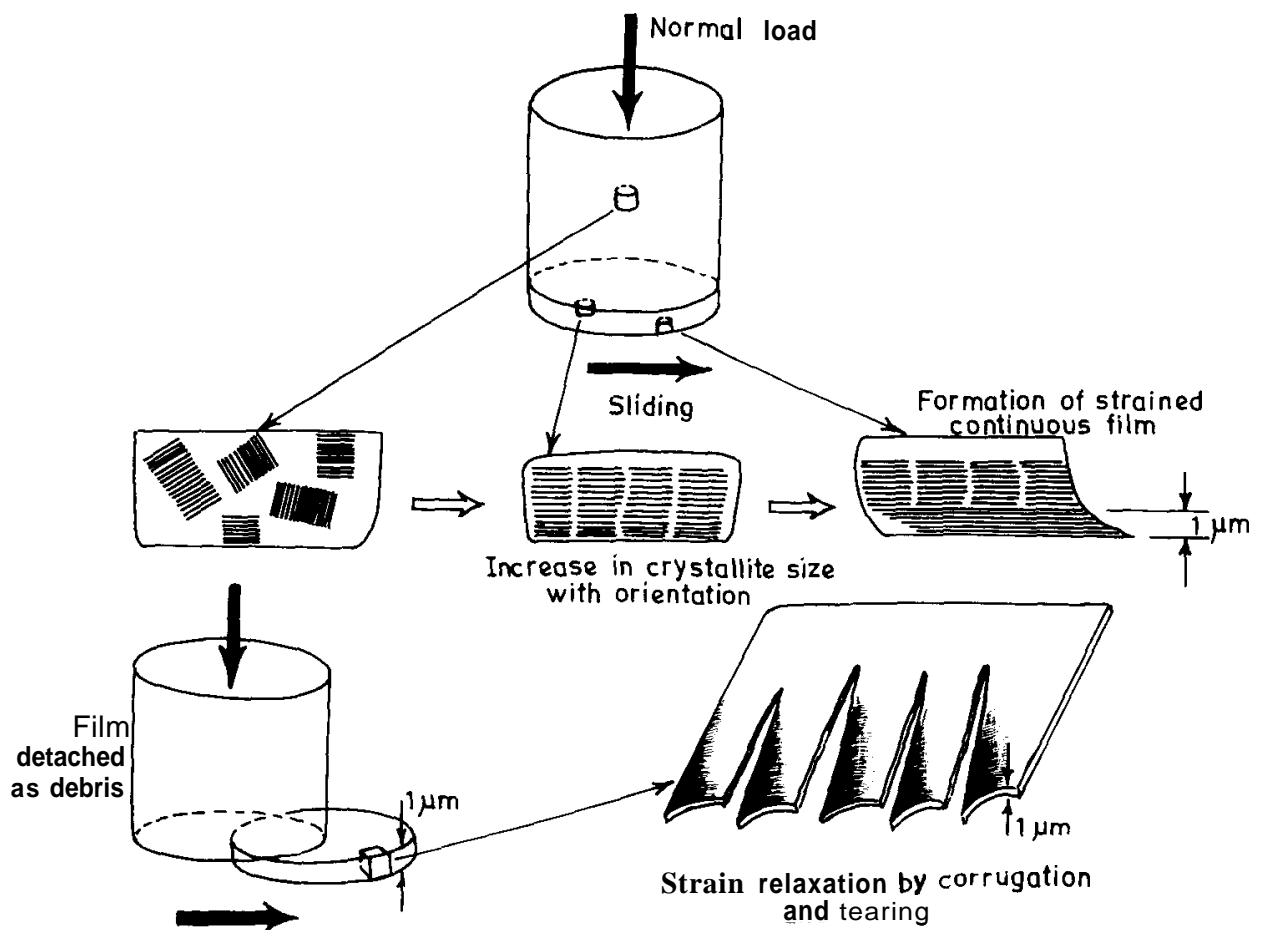

Figure 9 A schematic model of the wear of PTFE.

c dimensions (Table I) indicate that the effect of sliding on the unit cell dimensions is almost isotropic. The increase in the unit cell volume in the pretransition state could be attributed to an initial relaxation of the helical structure. The subsequent posttransition reduction in the unit cell volume is possibly due to improved molecular alignment within the unit cell. It may be pointed out that improved alignment of the flourine surfaces of neighbouring PTFE chains is likely to facilitate easy sliding of adjacent chains. As has been observed [IS] in the case of poly(p-phenylene terepthalamide), the shrinkage in the unit cell may have also contributed to the growth of crystallites, thus indirectly affecting the wear process.

Considering that the thickness of the debris was found to be of the order of $1 \mu \mathrm{m}$, the above model would imply the pressing together of anything up to SO $20 \mathrm{~nm}$-thick crystalline sheets. Initiation of the phenomena of crystallite growth would also mark the beginning of a high wear regime where these thick and large laminates shear against the bulk in giving rise to debris.

The laminates, as long as they are part of the PTFE specimen block, consist of highly strained material. Once they are detached from the bulk the strains are relaxed by warping, corrugation and tearing as seen in Fig. 3. However, the broadening of the reflections from the debris indicates that although the macrostresses developed during sliding may have been relieved, the microstresses in the grains persist even after the films detach from the bulk. The effect of the diminution in crystalline size on the profile broadening cannot also be ruled out.

The increased unit cell volume of the debris (determined from the X-ray diffraction pattern of the debris) suggests that the molecules perhaps return to a relaxed state similar to that observed in the pretransition state. The reduction in the intensity at high
$2 \mathrm{H}$ values also indicates a lack of long-range or threedimensional order of the structure. Further evidence for the reduction in three-dimensional order is obtained from the ratio

$$
\frac{\% \text { crystallinity of worn bulk }}{\% \text { crystallinity of worn debris }}=1.9
$$

estimated from the DSC endotherm at the transition (using the method given by Slade and Jenkins [16]); the molecular weight of PTFE has also been shown by Arkles and Schireson [17] to decrease by several orders of magnitude as it emerges as wear debris. A schematic model of the wear of PTFE based on the present observations is shown in Fig. 9. While further investigation is required to identify the agency of change in the wear rate during sliding, the change appears to be such as to bring about pronounced mechanical deformation in the thin layers adjacent to the worn surface. Strains are introduced and orientation of the crystallites changes sufficiently at the transition to increase crystallite size. These occur at a specific temperature which is sensitive to normal pressure and sliding speed and effect an irreversible change in the wear rate. The mechanical changes remain in the slid layer until such time as the strained layer is detached from the bulk by wear.

\section{Conclusions}

In sliding PTFE specimens against EN24 steel in the pressure range of 0.06 to $0.42 \mathrm{MPa}$ and sliding speed range of 0.1 to $I .2 \mathrm{~m} \mathrm{sec}$ " the wear rate was found to increase sharply at certain sliding distances. X-ray diffraction of the unworn and worn samples, and scanning electron micrographs of the debris, showed that sliding in the post-transition wear regime introduces (a) shrinkage of the unit cell, (b) enlargement of the crystallites and (c) no conformational changes. The presence of residual stresses in these layers adjacent to the sliding interface suggests that the 
crystallites, while still remaining integral to the bulk sample, may have been through a process of mechanical working. As soon as the surface layer becomes detached from the bulk as debris the residual stress and strains are released and the unit cell and crystallite attempt to achieve their original dimensions.

\section{Acknowledgement}

One of the authors (S.K.B.) is grateful to the Aeronautical Research and Development Board (India) for providing the necessary grant for this work. The authors are also grateful to Miss C. S. Lakshmi, Mr V. Kulkarni and Mr Ravishankar for their help during the course of this work.

\section{References}

1. K. R. MAKINSON and D. TABOR, Proc. R. Soc. $A 281$ (1964) 49.

2. C. M. POOLEY and D. TABOR, ibid. A329 (1972) 251.

3. K. TANAKA, Y. UCHIYAMA and S. TAYOOKA. Wear 23 (1973) 153.

4. K. G. MCLAREN and D. TABOR, Nature 197 (1963) 856.
5. N. G. MeCRUM, J. Polym. Sci. 34 (1959) 355

6. J. F. LONTZ and M. C. KUMNICK, Amer. Soc. Lubr. Eng. Trans. 6 (1963) 276.

7. B. J. BRISCOE and N. ZHILONG, Wear 100 (1984) 221.

8. H. A. RIGBY and C. W. BUNN, Nature 164 (1949) 583.

9. C. W. BUNN and E. R. HOWELLS, ibid. 174 (1954) 549.

10. E. S. CLARK and L. T. MUUS, Z. Krist. 117 (1962) 108.

11. L. E. ALEXANDER, "X-ray Diffraction Methods in Polymer Science" (Wiley, New York, 1969) p. 189.

12. D. W. Van KREVELEN, "Properties of Polymers" (Elsevier, Amsterdam, 1976) p. 386.

13. J. TOPPING, "Errors of Observation and Their Treatment" (Chapman and Hall, London, 1962) p. 73.

14. B. D. CULLITY, "Elements of X-ray Diffraction" (Addison-Wesley, Massachusetts, 1978) p. 447.

15. M. G. DOB B, "Handbook of Composites", Vol. 1 (Elsevier, Amsterdam, 1985) p. 700.

16. P. E. SLADE Jr and L. T. JENKINS, "Thermal Characterization Techniques" (Dekker, New York, 1970) p. 26.

17. B. C. ARKLES and M. J. SCHIRESON, Wear 39 (1976) 177

Received 23 April

and accepted 22 September 1987 